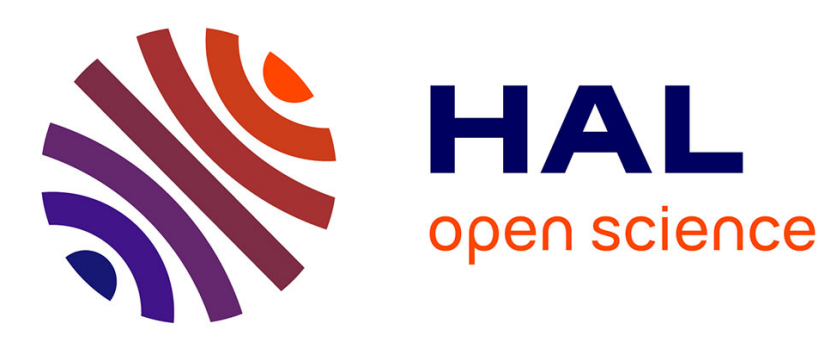

\title{
A Classification of flow-Stress Anomalies, and the Specific Role of Strain Rate Sensitivity
}

F. Louchet

\section{To cite this version:}

F. Louchet. A Classification of flow-Stress Anomalies, and the Specific Role of Strain Rate Sensitivity. Journal de Physique IV Proceedings, 1996, 06 (C2), pp.C2-189-C2-198. 10.1051/jp4:1996227 . jpa00254205

\section{HAL Id: jpa-00254205 https://hal.science/jpa-00254205}

Submitted on 1 Jan 1996

HAL is a multi-disciplinary open access archive for the deposit and dissemination of scientific research documents, whether they are published or not. The documents may come from teaching and research institutions in France or abroad, or from public or private research centers.
L'archive ouverte pluridisciplinaire HAL, est destinée au dépôt et à la diffusion de documents scientifiques de niveau recherche, publiés ou non, émanant des établissements d'enseignement et de recherche français ou étrangers, des laboratoires publics ou privés. 


\title{
A Classification of flow-Stress Anomalies, and the Specific Role of Strain Rate Sensitivity
}

\author{
F. Louchet
}

Groupe Physique du Métal, LTPCM (CNRS-URA 29), ENSEEG, Institut National Polytechnique de Grenoble, France

\begin{abstract}
We propose a classification of stress anomaly mechanisms based on two limiting cases, according whether the macroscopic behaviour directly reflects the individual dislocation properties, or involves the whole dislocation popalation. We show that, in the second case, some typical anomalies can be defined according to the relative efficiencies of mechanisms responsible for an increase or a decrease of the mobile dislocation density, and are associated with specific strain rate sensitivities. The way in which such an analysis can help in deciding which models are most relevant is discussed.
\end{abstract}

\section{INTRODUCTION}

A flow stress anomaly can be defined as a positive stress response to an increase of temperature at constant strain rate, instead of the usual negative one expected in thermally activated plasticity. This particular behaviout is observed in a wide variety of materials, including a number of ordered intermetallics, but also in some pure metals, despite their different crystallographic structures and dislocation microstructures. These materials most of ten exhibit other characteristic features as a strong work-hardening rate (WHR), a strain rate sensitivity (SRS) close to zero (usually associated with positive SRS transients), a partly reversible flow stress, and serrated stress-strain curves (strain bursts) (for a review, see [1]).

Among the available models proposed to date to account for these anomalies, some are based on an anomalous behaviour of the individual dislocation velocities, while others consider a possible evolution of the mobile dislocation density. Based on these two limiting cases, the main goal of the present paper is to propose an attempt of classification of stress anomalies, to discuss thoroughly their respective features, among which the strain rate sensitivity plays a central role, and to illustrate this classification by some models proposed for anomalies in $\mathrm{Ll}_{2}$ intermetallics, showing that it can help in deciding which of them are most relevant. The following discussion will implicitely refer to constant strain rate tests.

\section{2 . "V-TYPE" AND " $\rho$-TYPE" MECHANISMS}

\section{1 "v-type" mechanisms}

A possible reason for a strength anomaly is that the average velocity $V\left(\sigma^{*}, T\right)$ of individual dislocations has an anomalous temperature dependence, i.e. that $\left[\partial V\left(\sigma^{*}, T\right) / \partial T\right]_{\sigma^{*}}<0$ in a given temperature range, $\sigma^{*}$ being the effective stress. The stress response $\left[\partial V\left(\sigma^{*}, T\right) / \partial \sigma^{*}\right] \mathrm{r}$ of the dislocation velocity being usually positive, an increase of temperature has thence to be compensated by an increase of stress in order to keep the dislocation velocity constant. 
It must be noticed that, in this type of approach, the constant applied strain rate is considered to be equivalent to a constant dislocation velocity, which implicitely assumes that the mobile dislocation density is kept constant. The macroscopic behaviour of the material thus reflects directly the behaviour of individual dislocations. This is why this type of description can be considered as a 1-dislocation approach. Dislocation motion is hence necessarily steady, i.e. the average velocity of each dislocation has to temain constant with time.

\section{2 "p-type" mechanisms}

In contrast, if a possible variation of the mobile dislocation density is contemplated, the system is given an extra degree of freedom, and the motion of an individual dislocation must not necessarily be considered as steady. For instance, even if $V\left(\sigma^{*}, T\right)$ has a normal behaviour, mobile dislocations can be occasionally lost through an exhaustion mechanism, thus becoming sessile, or simply disappear through an annihilation process. In order to cope with the constant applied strain rate, the steady character of dislocation motion considered in "v-type" models has to be replaced in " $\rho$-type" ones by a steady state in $p_{\mathrm{m}}$ and $V$ (this is more or less equivalent to "v-type" approaches as long as the strain rate is kept constant, but essentially different as soon as strain rate changes are considered, as discussed later). In steady state conditions, the exhausted or arnihilated dislocations are replaced by new ones, for instance through multiplication. or through "remobilisation" of exhausted (and now sessile) dislocations. This balance between multiplication (or remobilisation) and exhaustion (or annihilation) terms is made necessary by the constant applied strain rate: if for instance the latter terms overwhelm the former, the mobile dislocation density is expected to fall off down to zero for a finite strain $\varepsilon_{0} \approx$ $\rho_{0} b A$ (o being the initial mobile dislocation density and $\boldsymbol{A}$ the mean free path of dislocations before exhaustion or annihilation). The flow stress would thus diverge at $\varepsilon_{0}$, which is usually not observed 1,2 .

The evolution with time of the mobile dislocation density $\rho_{\mathbf{m}}$ can be written:

$$
d \rho_{m}=\left[\left(-E \rho_{m}-A \rho_{m}^{2}+M \rho_{m}\right) V\left(\sigma^{*}, T\right)+R \rho_{s}\right] d t
$$

where $E, A, M$ and $R$ respectively stand for exhaustion, annihilation, multiplication and remobilisation "frequencies", and depend in principle on $\sigma^{*}$ and $T$, and where $\rho_{m}$ and $\rho_{s}$ are respectively the mobile and sessile dislocation densities. Both exhaustion and multiplication terms can be reasonably assumed to follow a first order kinetics in mobile dislocation density, since they usually involve a single mobile dislocation (multiplication occuring either at junctions between mobile dislocations and immobile forest dislocations, or at superjogs on a single mobile dislocation). In contrast, annihilation between two mobile dislocations follows a second order kinetics in $\rho_{\mathrm{m}}$, and remobilisation is likely to follow a first order kinetics in the sessile dislocation density $\rho_{\mathrm{s}}$. This is of importance for the following discussion.

The steady state mentioned above can be written $d \rho_{m}=0$, i.e.:

$$
\left[\left\{(M-E) \rho_{m}-A \rho_{m}^{2}\right\} \vee\left(\sigma^{*}, T\right)+R \rho_{s}\right]=0
$$

Exhaustion $^{3}$ and annihilation, involving either cross-slip or climb, are thermally activated. Their efficiencies are thus expected to increase with temperature. If they are more sensitive to temperature than multiplication or remobilisation, stress has to increase as temperature is raised, in order to fulfill the steady state condition of eq. (2), which obviously leads to a stress anomaly.

1 taking reasonable orders of magnitude for $\rho_{m}\left(10^{12} \mathrm{~m}^{-2}\right), \mathrm{b}\left(3 \times 10^{-10} \mathrm{~m}\right)$ and $\Lambda(1 \mu \mathrm{m})$, the stress would diverge for $\varepsilon_{c}=3 \times 10^{-4}$.

2 this steady state does not exclude the possibility of transients or instabilities, during which $\rho_{\mathrm{m}}$ and $V$ can fluctuate around their steady state values.

3 Athermal dislocation storage will be disre garded here. 


\section{3 . OTHER CONSEQUENCES ON MECHANICAL PROPERTIES (SRS AND WHR)}

\section{3 . 1 "v-type" mechanisms}

As defined in $\$ 2$, in pure "v-type" models, anomalies result from an anomalous temperature dependence of dislocation velocities, which must be compensated by a positive stress response of dislocation velocities in order to generate the anomaly. Since $\rho_{n}$ is assumed constant, the SRS of the material directly reflects the stress sensitivity of dislocation velocities. A strain rate jump yields an immediate readjustment of individual dislocation velocities, the stress response does not exhibit any transient, and, since $[\partial V / \partial \sigma]_{\mathrm{T}}>0$, the strain rate sensitivity is necessarily positive.

There is no specific provision in these "v-type" approaches for high work-hardening rates. since mobile dislocations have to remain mobile (steady motion) instead of being contimuously exhausted and stored as sessile dislocations, as considered in $\rho$-type approaches discussed now.

\section{2 " $\rho$-type" mechanisms}

\subsubsection{SRS}

In the case of " $\rho$-type" mechanisms, during a strain rate jump (e.g. increase of strain rate) at constant temperature, the material response is expected to take place in two steps. Starting from the steady state described above (eq. (2)), if $V\left(\sigma^{*}, T\right)$ has a normal stress behaviour, the stress suddenly increases at constant $\rho_{m}$, which changes in different ways the values of $E\left(\sigma^{*}, T\right)$,

$A\left(\sigma^{*}, T\right), M\left(\sigma^{*}, T\right)$ and $R\left(\sigma^{*}, T\right)$. The bracket thus departs from zero. A transient is then expected, during which $\rho_{m}$ and the corresponding stress evolve toward new steady state values, for which eq. (2) is again verified.

These two successive reactions can be described by two different types of SRS, a "transient" strain rate sensitivity $S_{t}=\partial \sigma^{*} / \partial \ln \dot{\varepsilon}$ and a "steady state" strain rate sensitivity $S_{S s}=\Delta \sigma^{*} / \Delta \ln \dot{\varepsilon}$. Since eq. (2) depends (at constant temperature) on both $\sigma^{*}$ and $\rho_{m}$, there is no reason why the new steady state stress should be the same as the initial one. The steady state SRS $\left(S_{\text {ss }}\right)$ generally differs from zero.

More precisely, combining Orowan's equation to eq. (2), the steady state condition can be written:

$$
-\frac{A}{V\left(\sigma^{*}, T\right)}\left(\frac{\dot{\varepsilon}}{b}\right)^{2}+(M-E) \frac{\dot{\varepsilon}}{b}+R \rho_{s}=0
$$

which can be easily resolved in $\dot{\varepsilon}$, giving:

$$
\dot{\varepsilon}=b \frac{V\left(\sigma^{*}, T\right)}{2 A}(M-E)\left\{1 \pm \sqrt{1+\frac{4 A R \rho_{S}}{V\left(\sigma^{*}, T\right)(M-E)^{2}}}\right\}
$$

from which the SRS can be in principle derived, if the stress dependencies of the different terms were known. General tendencies can nevertheless be found in a few particular cases, according to the relative magnitudes of these different terms.

\subsubsection{1 "ER-o-type" mechamisms}

In the case where annihilation and multiplication can be neglected in front of exhaustion and 
remobilisation, eq. (4) reduces to:

$$
\dot{\varepsilon}=b \frac{R \rho_{s}}{E}
$$

$R$ being usually more sensitive to stress than $E, \dot{E}$ is an increasing function of stress, and the SRS is positive (unless $R$ and $E$ have similar stress dependencies).

\subsubsection{2 "EM-0-type" mechanisms}

The dominant terms here are exhaustion and multiplication $(A=R=0)$. This is the basic assumption of the ELU model discussed in $\S 5.2 .2$ (and also of the LPU one proposed earlier for L1o alloys [2]). In this case, since the solution $\dot{\varepsilon}=0$ is not acceptable, eq. (4) leads to $E=M$ (eq. 3), which is only a function of stress and temperature. The imposed temperature determines the steady state stress (through eq. (3)), which means that the steady-state SRS is equal to zero. and the imposed strain rate determines the mobile dislocation density (through the Orowan equation).

Pure "EM- $\rho$-type" mechanisms are thus expected to exhibit a non zero (positive) $S$ and a zero $S_{\text {ss. }}$

\subsubsection{3 "EMA-p-type" mechanisms}

This is what would happen if some annihilation is allowed to take place in a "EM- $\rho$-type" mechanism. Again, since $\dot{\varepsilon}=0$ is not acceptable, eq. (4) gives:

$$
\dot{\varepsilon}=b \frac{(M-E) V\left(\sigma^{*}, T\right)}{A}
$$

In order to keep $\dot{\varepsilon}$ positive, multiplication must obviously be more efficient than exhaustion. The SRS is in general different from zero, and, since multiplication is likely to be more stress sensitive than exhaustion or annihilation, the SRS should be positive. It is worth noticing that, at constant strain rate, the steady state condition $E=M$ (eq. 3) is obtained at vanishing values of $A$, corresponding to a zero SRS.

\subsubsection{WHR}

The steady state described above is a steady state for the mobile dislocation density. The sessile dislocation density is expected to increase continuously, due to the exhaustion mechanism, though somewhat tempered by annibilation. This can result in a strong work-hardening rate, through an internal stress:

$$
\sigma_{i}=\alpha u b \sqrt{\rho_{s}+\rho_{m}}
$$

in which the sessile dislocation density continuously increases with strain [2].

In the case of "EM $\rho$-type" mechanisms, exhaustion prevails upon annihilation, and remobilisation is negligible. Storage of sessile dislocation is thus enhanced, and the WHR is expected to be even larger than in the case of "ER- $\rho$-type" or "EMA- $\rho$-type"mechanisms. This might be the reason why, in $\mathrm{L}_{2}$ compounds in particular, the WHR usually goes through a maximum at a temperature for which the SRS is minimum, i.e. below the stress peak temperature. The mechanism responsible for the anomaly might indeed evolve from a nearly pure "EM $\rho$-type" at the SRS minimum to a more general " $\rho$-type" one as temperature approaches the stress peak temperature.

Other properties often associated with stress anomalies, as for instance flow stress reversibility, can also be derived from such "EM $\rho$-type" approaches (e,g. [2,3]). 


\section{COMPETITION BETWEEN ZERO SRS AND NON-ZERO SRS MECHANISMS}

It appears from the above discussion that "EM- $\rho$-type" mechanisms are characterised in particular by a zero SRS, which is usually observed experimentally, whereas this is not necessarily the case of general " $\rho$-type" nor "v-type" ones. Does that mean that the latter are irrelevant? This question deserves more attention. This is why we shall focus now on the way in which a mechanism with a zero SRS and a mechanism with a non-zero SRS, acting in parallel, compete in the material ${ }^{4}$.

Taken alone, these mechanisms are characterised respectively by flow stresses $\sigma_{0}^{*}(T)$ and $\sigma_{1}^{*}(T, \dot{\varepsilon})$, the first one depending on $T$ only since the corresponding SRS is zero. Due to the usual complexity of the crystallographic structures in intermetallics, several types of dislocations might be involved in deformation processes. The result of the competition between mechanisms 0 and 1 depends on whether they involve the same type or different types of dislocations, as examined now.

\subsection{The two mechanisms involve different types of dislocations}

It is obvious that, since $\sigma_{0}^{*}(T)$ is independent of $\dot{\varepsilon}$, and since $\sigma_{1}^{*}(T, \dot{\varepsilon})$ is an increasing function of $\dot{\varepsilon}$, the easiest will be mechanism 0 at high strain rates, and mechanism 1 at low strain rates.

At a given temperature indeed, the steady state of mechanism 0 ("EM- $\rho$-type") is determined by a single value $\sigma_{0}^{*}(T)$ of stress, as seen in $\$ 3.2 .1 .2$ and eq. (3).

- At large strain rates, $\sigma_{0}^{*}(T)<\sigma_{1}^{*}(T, \dot{\varepsilon})$, and mechanism 0 ("EM-p-type"), whose steady state stress is lower, is dominant, i.e. gives a major contribution to the total strain (fig. 1).

- At low strain rates, $\sigma_{0}^{*}(T)>\sigma_{1}^{*}(T, \dot{\varepsilon})$, and mechanism 1 , which has a positive SRS, turns to be the easiest. The resulting effective stress is lower than $\sigma_{0}^{*}$, which enhances the exhaustion kinetics of the "EM- $\rho$-type" mechanism as compared to its multiplication rate. Mobile dislocations of the "EM- $\rho$-type" become gradually sessile, and mechanism 1 eventually operates alone.

\section{2 B oth mechanisms involve the same dislocations}

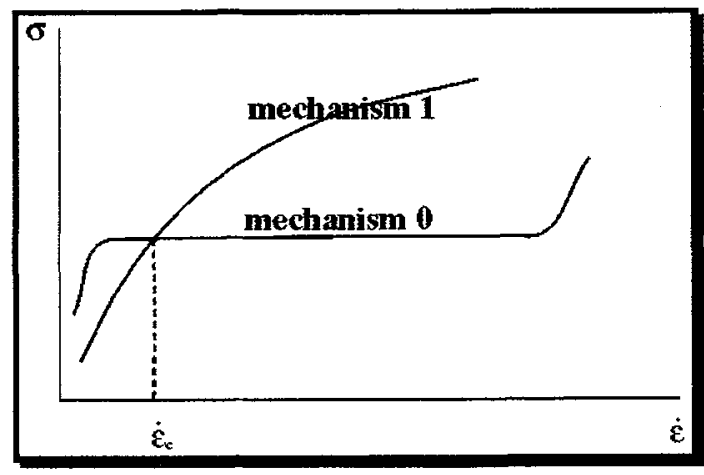

4 both mechanisms can be either normal or anomalous. 
Figure 1: Typical strain rate dependence of the flow stress for mechanisms of types 0 and 1, at constant temperature. Mechanism 1 with a positive SRS, can become dominant at low strain rates $\left(\dot{\varepsilon}<\dot{\epsilon}_{c}\right)$.

- At large strain rates, $\sigma_{1}^{*}(T, \dot{\varepsilon})>\sigma_{0}^{*}(T)$ : the "EM- $\rho$-type" mechanism is and remains dominant as in the previous case.

- At low strain rates, $\sigma_{1}^{*}(T, \dot{\varepsilon})<\sigma_{0}^{*}(T)$ : the flow stress starts close to $\sigma_{1}^{*}$, and the activity of mechanism 1 starts being larger than that of mechanism 0 , while the exhaustion due to mechanism 0 ("EM- $\rho$-type") prevails upon multiplication since the stress is lower than $\sigma_{0}^{*}$. This would readily eliminate all the mobile dislocations, which is not possible in an imposed strain rate test. Stress has therefore to increase up to $\sigma_{0}^{*}$, which restores a balance between multiplication and exhaustion, and thence a significant activity of the "EM- $\rho$-type" mechanism. Both mechanisms could operate simultaneously in this case, but at a stress level imposed by the "EM-p=type" process. In particular, a strain rate jump would lead to a change of density of mobile dislocations, leaving unchanged the steady state flow stress, and a zero steady state SRS would be measured after the transient.

To summarise, an "EM- - -type" mechanism in parallel with a mechanism with a positive steady state SRS will always be active, if not dominant, except if the two mechanisms involve different types of dislocations and if at the same time the strain rate is low enough In the case where the same dislocations are involved in the two mechanisms, the anomaly appears, with a zero SRS, whatever the normal or anomalous character of the competing mechanism. This is probably the reason why most anomalies show a zero (or small) value of $S_{s s}$.

\section{EXAMPLE: THE FLOW STRESS ANOMALY OF L12 ALLOYS}

\section{1 "v-type" models}

\subsection{I "local pinning" models}

The first models proposed for flow stress anomalies in $\mathrm{L}_{2}$ alloys were based on a thermally activated pinning of superdislocations $[4,5]$. The pinning rate thus increases with temperature, leading to a decreasing separation between pinning points. If the mobile dislocation density is assumed constant during deformation, the steady character of dislocation motion involves that the unpinning rate has to increase with temperature, which in turn requires an increase of stress. We have seen above that these models do not in principle systematically account for a zero SRS. However, the introduction of a thermally activated unpinning [6] yields a logarithmic dependence of stress on strain rate, i.e. a low SRS, whereas considering the possibility of different paths from the pinned state to the unpinned one, combined with the same steady state assumption, allows a good description of the discontinuities of the activation volume [7].

\subsection{2 "locking-unlocking" (L/UL) model}

This type of model is based on in situ observations, performed on different types of materials exhibiting stress anomalies, of the jerky motion of screw dislocations [8]. This well known approach has been applied to a number of different materials, and will be briefly sketched here. Its basically consists in a series of thermally activated locking and subsequent unlocking events experienced by screw superdislocations, related (in $\mathrm{L} 1_{2}$ alloys) to cross-slip from the octahedral towards the cube plane, which account for the jerky motion observed during in situ straining in a transmission electron microscope.

Both locking and unlocking frequencies, defined through the corresponding activation energies 
$\Delta G_{L}$ and $\Delta G_{U L}$, increase with temperature. The waiting time in the locked configuration is observed to be always significantly larger than the flight time between two successive lockings. This means that $\Delta G_{U L}-\Delta G_{L}$ is positive, and that the average dislocation velocity, given by:

$$
V\left(\sigma^{*}, T\right) \propto \exp \left[-\frac{\Delta G_{u L}-\Delta G_{L}}{k T}\right]
$$

has a normal temperature behaviour, unless some additional ingredient is introduced. In its last developments, the authors consider that the unlocking mechanism requires a stress which increases with the amount of cross slip into the cube plane, i.e. with temperature. As temperature is raised, average dislocation velocities decrease, unless stress is raised to keep $V\left(\sigma^{*}, T\right)$ constant.

This is a typical one-dislocation "v-type" model. The SRS has to be positive, as for instance in the case of the local pinning models. It can be nevertheless noticed that the L/UL mechanism could be described in a more realistic way by an "ER- $\rho$-type" model rather than by a pure " $v$ type" one. The SRS would be anyway positive (see $\$ 3.2 .1 .1$ ).

\section{2 " $\rho$-type" models}

\subsubsection{Greenberg-Ivanov}

Though the Greenberg-Ivanov approach [9] can be (and has been) applied to stress anomalies in intermetallics, it is more general, and aims at relating the macroscopic behaviour of a material to the evolution of the different possible populations of sessile and glissile dislocations. In its simplest form, it considers the time evolution of a single type of mobile and sessile dislocation densities:

$$
\begin{aligned}
& \frac{d \rho_{m}}{d t}=-\rho_{m} \nu_{m s}+\rho_{s} v_{s m}+M^{\prime} \\
& \frac{d \rho_{s}}{d t}=\rho_{m} v_{m s}-\rho_{s} v_{s m}
\end{aligned}
$$

where $\rho_{m}$ and $\rho_{s}$ are respectively the mobile and sessile densities, where $\nu_{m s}$ and $\nu_{s m}$ are respectively the frequencies for the $m->s$ and $s->m$ transformations, and where $M^{\prime}=d \rho / d t>0$ is a multiplication rate for the total dislocation density $\rho=\rho_{m}+\rho_{s}$ (it can be noticed that eq. (10) is rather similar to eq. (1). These equations are made complete by the addition of two well known other equations relating the microscopic and the macroscopic behaviours:

$$
\begin{aligned}
& f \sigma=\alpha \mu b \sqrt{\rho_{s}+\rho_{m}} \\
& \dot{\varepsilon}=\rho_{m} b f V
\end{aligned}
$$

where $f$ is the Schmid factor, and $V$ the instantaneous velocity of the mobile dislocations.

When the "observation time" is large as compared to the "relaxation time" $\left(v_{m s}+y_{s m}\right)^{-1}$, eq. (6) becomes quasi-stationnary, as assumed in the above mentioned " $p$-type" models. If $v_{m s} \gg v_{s m}$ (which is usually the case), and provided some other (and more questionable) assumptions are made (as for instance that both $V$ and $M^{\prime}$ are stress independent), an analytical solution can be found for $\sigma(T, \dot{\varepsilon})$, which goes through a maximum as temperature is raised.

This model is clearly a " $\rho$-type", but not an "EM- $\rho$-type" one. It does not give in principle a zero SRS, for at least two reasons:

i) in eq. (10) the "remobilisation" term $\left(\rho_{s} v_{s n}\right)$, which is independent of $\rho_{m}$ (as the exhaustion term does), is not neglected, and that the multiplication term $M^{\text {' }}$ is taken independent of $\rho_{\mathrm{m}}$, in contrast with eq. (1) where $M^{\prime}=M V\left(\sigma^{*}, T\right) \rho_{m}$. As stated above, the multiplication term should in principle depend linearly on $\rho_{m}$, since a single mobile dislocation is involved in most multiplication mechanisms.

ii) the stress appears only in eq. (12), as an internal stress due to the long range 
interactions of mobile dislocations with the total dislocation population. It means that, though a Schmid factor is introduced in eq. (13), the effective stress which rules both the velocity $V\left(\sigma^{*}, T\right)$ of mobile dislocations and the transition frequencies $\nu_{m s}$ and $\nu_{s m}$ is ignored.

5.2.2 The "Extended-Locking/Unzipping" (ELU) model

This model [10] is a typical "EM- - -type" model, based on macrokink mechanisms similar to those involved in the Chrzan-Mills simulation [11], and, as the Greenberg-Ivanov one, on a steady state assumption that avoids a complete exhaustion or a catastrophic multiplication of dislocations. A thermally activated locking of superdislocations into KW locks leads to an exponential macrokink height distribution:

$$
n(\lambda)=n_{0} \exp \left[-v_{0} \exp \left(-\frac{\Delta G_{2}}{k T}\right) \frac{\lambda}{\nu\left(\sigma^{*}, T\right)}\right]=n_{0} e^{-\lambda / \lambda_{0}}
$$

MKs experience a friction $F_{0}$ (essentially due to bending of $\mathrm{KW}$ locks in the cube plane, and supposed to be constant for the sake of simplicity), and can therefore zip along $\mathrm{KW}$ locks if their heights $\lambda$ are larger than a critical height $\lambda_{P}=F_{0} / \sigma^{*} b$. If a dislocation is in such a configuration that it has at least one MK larger than $\lambda_{P}$, this MK will zip, react with other MKs, and give continuously new configurations until one of them has no MK larger than $\lambda_{P}$. This configuration is sessile, and the occurence of such events in the mobile dislocation population is at the origin of exhaustion. The related exhaustion rate $E\left(\sigma^{*}, T\right)$ can be computed from the height distribution $P(\lambda)$ of the largest MK on each dislocation, which can be shown easily to be:

$$
P(\lambda)=n(\lambda)\left[1-\frac{\int_{\lambda}^{\infty} n(\lambda) d \lambda}{\int_{0}^{\infty} n(\lambda) d \lambda}\right]=n_{0} e^{-\lambda / \lambda_{0}}\left[1-e^{-\lambda / \lambda_{0}}\right]
$$

The exhaustion rate is then:

$$
E\left(\sigma^{*}, T\right) \propto \int_{0}^{\lambda_{p}} P(\lambda) d \lambda
$$

which depends on stress through $\lambda_{P}$ and on temperature through $P(\lambda)$.

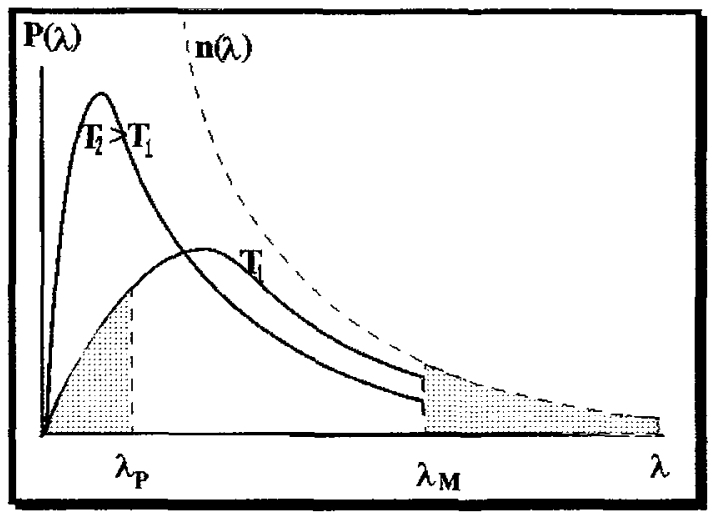

Figure 2: schematic macrokink heights distributions $\mathrm{n}(\lambda)$ and $\mathbf{P}(\lambda)$ for two temperatures (see text). The grey areas correspond to the exhaustion and multiplication frequencies at $T=T_{1}$.

The steady state assumption requires that this exhaustion rate is balanced by multiplication, annihilation being neglected in the model. It is assumed, as in other MK models, that MKs heigher than a critical height $\lambda_{M} \approx \mu b / \sigma^{*}$ are potential dislocation sources. The related multiplication rate is thus

$$
M\left(\sigma^{*}, T\right) \propto \int_{\lambda_{1 S}}^{\infty} n(\lambda) d \lambda=n_{0} \lambda_{0} e^{-\lambda_{A S} / \lambda_{0}}
$$

which depends on stress through $\lambda_{M}$ and on temperature through $\lambda_{0}$. 
The steady state requires that $E=M$. The anomaly arises from the fact that, as temperature is raised, the distributions $n(\lambda)$ and $P(\lambda)$ are shifted towards smaller $\lambda$ values (fig. 2), and a shift of $\lambda_{P}$ and $\lambda_{M}$ toward the left, i.e. an increase of stress, is needed to maintain the balance between multiplication and exhaustion. The other consequences of the model are those of \$3.2.1.2.

Due to its "EM- $\rho$-type" character, this model accounts qualitatively for the major observed features, namely a zero SRS, a significant flow-stress reversibility and a high WHR, in spite of the rather crude assumptions on which it is based.

\subsection{Which type of model is most relevant?}

We have given a few examples of a number of models proposed to account for the anomalous behaviour of $\mathrm{L} 1_{2}$ alloys. The question arises now on which type of model is most relevant, and will be discussed on the example of L/UL and ELU processes.

If the "local pinning" and the Greenberg-Ivanov approaches are discarded for the reason that the corresponding microstructures are either not in agreement with observations or not specified, we can focus the discussion on the L/UL mechanism (which should be better described by an "ER- $\rho$-type" model rather than by a "v-type" one) and a "EM- $\rho$-type" one like ELU, the latter only being characterised by a zero SRS. The locking-unlocking mechanism is actually observed in situ, and the question arises whether it can operate in the bulk as well. Both mechanisms apparently involve the same dislocations. However, the exhausted dislocations of the ELU mechanism are not sessile with respect to the L/UL one. In other words, the exhaustion of superdislocations taking place during the operation of the ELU mechamism cannot hinder a possible activity of the competing L/UL mechanism, and the conclusions of $\$ 4.1$ can then apply, instead of those of $\$ 4.2$. It turns out from the above discussion that the L/UL "v-type" or "ER- $\rho$-type" process can operate significantly at low strain rates, wheras the ELU "EM-p-type" one is likely to prevail at higher strain rates. Since the SRS is very small (for usual strain rates) in the whole anomaly temperature range, an "EM-o-type" model (like ELU) is more likely to be relevant in these conditions.

Since the L/UL mechanism might become dominant at lower strain rates, SRS measurements would give in this case positive values, but one can wonder whether the anomaly would still be observed. It cannot probably be inferred that L/UL is anomalous from in situ local stress measurements performed at usual strain rates [12], which exhibit a stress anomaly, since the stress measured in the thin parts of the specimen can reflect the characteristics of the mechanisms acting simultaneously in the bulk. The question whether the anomalous character of the L/UL mechanism is relevant or not is therefore still open. An experimental evidence of an anomaly associated with a positive SRS at low strain-rates should perhaps give an answer.

\section{CONCLUSION}

In the present analysis, stress anomalies have been classified into "v-type" and several " $\rho$-type" ones, which can be respectively described as "one-dislocation" and "multi-dislocation" processes. The imposed constant strain rate requires a steadiness of the individual dislocation motion in the former case, and a steady state in mobile dislocation density in the latter. Since the locking processes involved in both types of approaches are similar, in that they essentially involve dislocation core structures, some of the related particular features usually associated with stress anomalies, as for instance violations of the Schmid law, can be accounted for by both types of approaches. However, other specific properties, as a high work-hardening rate, a significant flow stress reversibility, and, most important, a low strain rate sensitivity associated with different possible types of strain rate jump transients, cannot be systematically derived 
from "v-type" approaches nor from general " $\rho$-type" ones. In contrast, these features come out in a natural way from "EM-p-type" mechanisms, in which exhaustion and multiplication are dominant. A possible competition of these two kinds of processes simultaneously operating in the material has been thoroughly discussed. Such an analysis can probably help in deciding to which type of mechanism the observed stress anomalies should be ascribed.

Nevertheless, most stress anomalies are also associated with strain bursts probably responsible for both dislocation avalanches and serrated flow at respectively the microscopic and the macroscopic scales. Though such features can be reasonably expected for small (or negative) strain rate sensitivities, none of the models proposed to date can account for it. The " $\rho$-type" approaches, which are given an extra degree of freedom through the possibility of varying the dislocation densities and thus describing strain rate transients, are obviously the basic framework in which further investigations of the relations between stress anomalies and plastic instabilities could be envisaged. The necessary (and difficult) ingredient to be introduced however is the particularly enhanced dislocation interactions, due the localised character of slip inherent in these phenomena, transforming the "multi-dislocation" approaches discussed here into "many-dislocation" ones, as already done in other domains.

\section{Aknowledgements}

The author is indebted to Dr J. Bonneville for comments on the manuscript.

\section{References}

[1] Veyssière P., and Saada G., 1995, in "Dislocations in Solids", vol. 10, F.R.N. Nabarro ed., to be published.

[2] Louchet F. and Viguier B.,Phil. Mag. A 71, n'6 (1995), 1313 - 1312.

[3] Louchet F., Phil. Mag. A (1995), in press, 1017 - 1024.

[4] Takeuchi S, and Kuramoto E, Acta Met. 21, (1973), 415 - 425.

[5] Paidar V., Pope D.P., and Vitek V., Acta Metall Mater.32, No 3 (1984), 435 - 448.

[6] Vitek V. and Sodani Y.,Scripta Metall. Mater. 25, (1991), 939 - 944.

[7] Khanta M., Cserti J, and Vitek V., a) Scripta Metall. Mater. 27 (1992), 481 - 486, b) Scripta Metall. Mater. 27 (1992), 487 - 492.

[8] Farenc S., Caillard D. and Couret A., Acta Metall. Mater. (1995) to be published.

[9] Greenberg B.A. and Ivanov M.A.,Mat. Sci. Engn. A153 (1992) 356 - 363.

[10] Louchet F., Journal de Physique III France (1995), in press.

[11] Mills M.J. and Chrzan D.C., Acta Metall. Mater. 40, No 11, (1992), 3051 - 3064.

[12] Couret A. and Caillard D.,Phil.Mag. A 59 (1989), 801 - 819. 